\title{
Extraction of Vegetation Image Index Using Normalized Difference Vegetation Index Algorithm
}

\author{
Dr. Ace C. Lagman ${ }^{1+}$, Joferson L. Bombasi ${ }^{2}$ and Chul-Soo Ye, Ph.D ${ }^{3}$ \\ ${ }^{1}$ FEU Institute of Technology \\ ${ }^{2}$ FEU Institute of Technology \\ ${ }^{3}$ Far East University Korea
}

\begin{abstract}
The study aims to create an application using openCV that can identify and calculate the vegetation part of a satellite image. The study utilized the two out of seven Landsat- 8 band images over a part of Metro Manila in Philippines acquired on Feb. 13, 2016. These band images are Bands 4 and 5 which are normally used to compute the vegetation index of a satellite image.

The algorithm calculates the relative area of the vegetation using Normalized Difference Vegetation Index or NDVI formula. The output of the NDVI creates a single-band dataset that only shows greenery. Values close to zero represent rock and bare soil and negative values represent water, snow and clouds. Taking ratio or difference of two bands makes the vegetation growth signal differentiated from the background signal. Water has an NDVI value less than 0 , bare soils between 0 and 0.1 , and vegetation over 0.1 . Increase in the positive NDVI value means greener the vegetation.

The sample satellite image is Manila with Landsat- 8 operational land imager (OLI) images. The study aims to test the NDVI formula in extracting vegetation index of Manila region which can be used to monitor the urban and classification of a certain region.
\end{abstract}

Keywords: remotes sensing, ndvi, satellite image

\section{Background of the Study}

Index images such as normalized difference vegetation index (NDVI) (Rouse, 1974). An index image is generally obtained using two or more band images and is adopted as a band image representing a specific attribute. In this paper, the researchers calculated the vegetation part of the satellite images using NDVI Algorithm.

The satellite images of vegetation show the density of plants. These remote sensing images are divided into regions - visible, infrared and microwave. The visible regions are further subdivided into bands such as blue, green and red. These satellite data are transformed into vegetation indices or values using formulas or algorithms to describe the density or greenness of vegetation.

The most common formula or measurement used to transform satellite raw data into values which can be used to calculate the density of vegetation is Normalized Difference Vegetation Index or NDVI. NDVI values range from +1.0 to -1.0 . Very low values of NDVI ( 0.1 and below) correspond to barren areas of rock, sand, or snow. Moderate values represent shrub and grassland ( 0.2 to 0.3$)$, while high values indicate temperate and tropical rainforests ( 0.6 to 0.8 ).

Using image analysis, vegetation can be classified as either green or stressed vegetation. The relative amount of vegetation for one specific date or between two or more dates can be determined by comparing the near infrared bands and red bands from the satellite image.

\footnotetext{
+ Corresponding author.

E-mail address: aclagman@feutech.edu.ph.
} 


\subsection{Research Questions}

1. How to create an image loading and writing for vegetation class?

2. How to use the NDVI algorithm in classifying a vegetation class?

3. How to test the accuracy of the identified and calculated region of vegetation from the satellite image?

\section{Scope and Limitation}

The study focuses in identification and calculation of vegetation from a satellite image which focuses on Manila Area. Using remote sensing, the satellite image of vegetation will be processed to show the density of plants. The relative amount of vegetation can also be calculated using NDVI and through image analysis, vegetation can further be classified as green or stressed vegetation.

The study utilized the two out of seven Landsat- 8 band images over a part of Metro Manila in Philippines acquired on Feb. 13, 2016 (Figure 2). These bond images are Band 4 and Band 5 which are normally used to compute the vegetation index of a certain satellite image.

The application has three features, which are image loading, image writing and NDVI calculation which consist of the following procedures image loading, image writing and calculation of Normalized Difference Vegetation Index calculation.

\section{Literature Review}

Mandrake et al. (2012) mentioned that remote sensing provides a powerful way to detect presence of surface sulfur deposits at the Borup Fiord glacial springs in Canada. In the study Surface Sulfur Detection via Remote Sensing conducted by Lukas Mandrake Et al. and published in ACM journal in the year 2012, a data-driven machine learning solution was created to evaluate methods that will identify the appropriate spectral wavelengths needed for sulfur detection. In this study, the sulfur in the Borup Fiord glacial spring was found on ice and rock.

Varun et al. (2011) mentioned that Enhanced Vegetation Index (EVI) and Fraction of Photosynthetically Active Radiation (FPAR) are two remote sensing datasets measured by Moderate Resolution Imaging Spectro-radiometer (MODIS) algorithms in NASA's Terra satellite. These were used in the study Monitoring Global Forest Cover using Data Mining by. The EVI measures the amount of vegetation at a particular location while FPAR measures photosynthesis. Segmentation-based algorithms have been used to distinguish if a land cover undergone change or not.

Horn, B., and Bachman, B. (1978) discussed that classification of terrain using satellite imagery requires exact alignment of edges and curves of such image with digital terrain models to deal correctly with the effects of varying sun angle and surface slope. Alignment is achieved by matching the real image with synthetic image obtained from a surface model and known positions of the light sources. The reluctance map is used to calculate the intensity of the synthetic image which accounts for self-shadowed surfaces but not shadows cast by one surface element on another. Berthold Horn and Brett Bachman matched the real image and synthetic images using similarity measure and search technique as discussed in the article

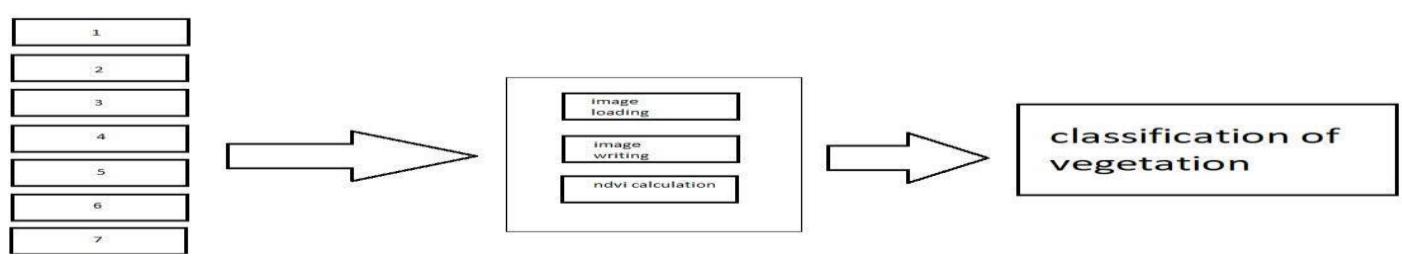

Fig. 1: Conceptual Framework

As test images, we used seven Landsat- 8 band images over a part of Metro Manila in Philippines acquired on Feb. 13, 2016 (Figure 2). The researchers created one index image for NDVI. 
For image classification, the researchers only used the bond 4 and 5 of Landsat- 8 seven band images and one index image. The following procedures were performed by the researcher using OPEN CV. These procedures are image loading, writing and NDVI calculation.

\section{Research Methodology}

The study utilizes remote sensing and image processing and analysis.

\section{A. Image Loading}

In this phase, images taken from the satellite is loaded as an input for the system. The image is preselected based on the needed bands for the computation.

\section{B. Image Writing}

In this phase, the resulting image after applying the NDVI calculations on the NIR and RED band images, is saved to a location

\section{NDVI Calculation}

NDVI is a graphical indicator used to analyze remote sensing measurements. It is calculated based on the reflection of the light through the visible and near-infrared wavelengths of satellite images which can be observe in the NIR and RED bond. The formula for NDVI is as follows:

$$
N D V I=\frac{(N I R-R E D)}{(N I R+R E D)}
$$

Eq. 1: NDVI Formula

\section{Red}

DN values from RED band

E. NIR

DN values from Near Infrared band

\section{Results and Discussion}

Answering Research Question No.1 entails the researcher to load and to write satellite images. The researcher used the NIR and Red Bonds images. The Near Infrared (NIR) is a subset of the infrared band of the electromagnetic spectrum, covering the wavelengths ranging from 0.7 to 1.4 microns. Based on the formula for NDVI, the following procedures were used to compute for NDVI extraction (i) Adding of NIR and RED Image for NDVI Calculation (ii) Subtraction of NIR and RED Image Calculation.

The following procedures were used for image writing.

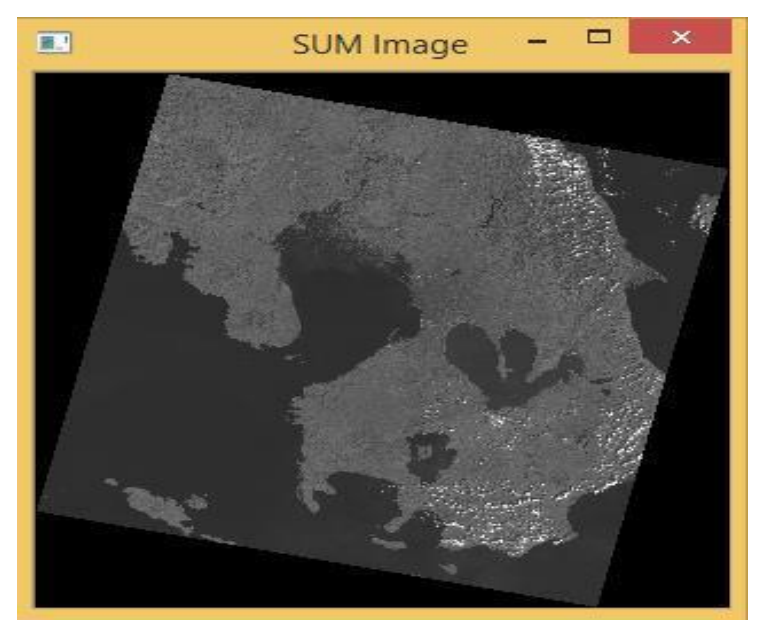

Fig.2 : Adding of NIR and RED Image for NDVI Calculation 


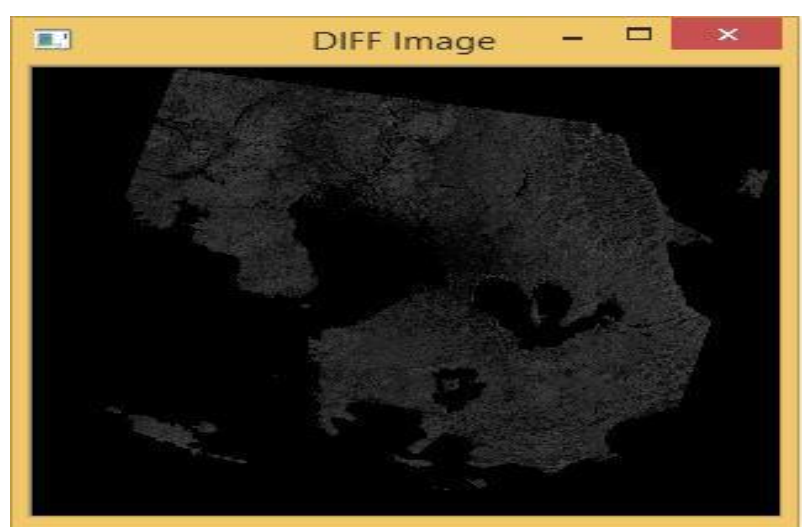

Fig. 3 : Subtractions of NIR and RED Image Calculation

Answering Research Question No.2 entails the researchers to compute for the vegetation area using the satellite images. To determine the vegetation, NDVI formula was used.

The result of the NDVI calculation for a given pixel ranges from -1 to +1 . To assess the computed NDVI results, the following legends for different cover types were used. The cover type consists of dense vegetation, dry bare soil, clouds, snow and ice and water.

Table 1: NDVI Calculation Legend

\begin{tabular}{|c|c|c|c|}
\hline COVER TYPE & RED & NIR & NDVI \\
\hline Dense vegetation & 0.1 & 0.5 & 0.7 \\
\hline Dry Bare soil & 0.269 & 0.283 & 0.025 \\
\hline Clouds & 0.227 & 0.228 & 0.002 \\
\hline Snow and ice & 0.375 & 0.342 & -0.046 \\
\hline Water & 0.022 & 0.013 & -0.257 \\
\hline
\end{tabular}

Table shows typical reflectance values in the red and infrared channels, and the NDVI for typical cover types. Water typically has an NDVI value less than 0 , bare soils between 0 and 0.1 and vegetation over 0.1 .

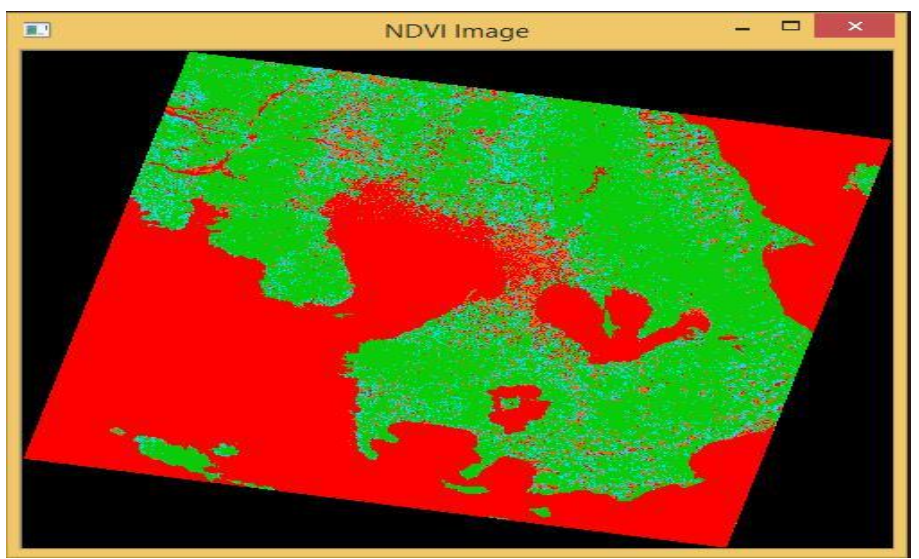

Fig.4 : Image Screenshots After Using NDVI Formula

Figure 2.0 reveals that after processing the two satellite bonds using NDVI formula, the results indicate that pixel values greater than $>0.3$ and less than 0.6 are considered vegetation area which are clearly shown in the image provided above.

Answering Research Question No. 3 entails the researchers to compare the NDVI calculated values to the pixel values of the green portion of the equivalent visible light image (Bands 3,2,1) of the LANDSAT-8 satellite image.

\section{Conclusion}


This paper presents a method to extract the vegetation part of a satellite image. This method will be very beneficial in conducting similar researches particularly in land monitoring. The formula of NDVI clearly identifies vegetation class. The following methods were used to calculate the NDVI results which are image loading, and image writing. Based on the formula for NDVI, the following procedures were used to compute for NDVI extraction (i) Adding of NIR and RED Image for NDVI Calculation (ii) Subtraction of NIR and RED Image Calculation.

\section{Future Works}

The study aims to include the normalized difference water index (NDWI) and normalized difference built-up index (NDBI). The computation of NDVI, NDWI and NDBI from Landsat-8 operational land imager (OLI) can lead to much accurate results. The study aims to test the image indexes using an image data to clearly identify the miss classification results of the three image indexes.

\section{References}

[1] Rouse, J.W., R.H. Haas, J.A. Schell, and D.W. Deering,(1974). Monitoring vegetation systems in the Great Plains with ERTS, Third Earth Resources Technology Satellite Symposium, Vol. 1, pp. 309-317.

[2] Mandrake, L., Rebbapragada, U., Wagstaff, K., Thompson, D., Chien, S., Tran, D., Pappalardo, R., Gleeson, D., and Castano, R., (2012). "Surface sulfur detection via remote sensing and onboard classification". ACM: New York

[3] Horn, B., and Bachman, B. (1978). "Using synthetic images to register real images with surface models". ACM: New York.

[4] Lasaponara, Rosa and Masini, Nicola (2012). "Satellite Remote Sensing".Springer:New York.

[5] Mithal, V., Garg, A., Boriah, S., Steinbach, M., Kumar, V., Potter, C., Klooster, S. and Castilla-Rubio, J. (2011). "Monitoring global forest cover using data mining". ACM: New York.

[6] Jones, Hanalyn G. and Vaughan, Robin A (2010). "Remote Sensing of Vegetation". Oxford University Press: New York.

[7] Chuvieco, Emilio and Huete, Alfredo (2009). "Fundamentals of Satellite Remote Sensing". CRC Press:Florida 